\title{
Calculus of convex polyhedra and polyhedral convex functions by utilizing a multiple objective linear programming solver ${ }^{1}$
}

\author{
Daniel Ciripoi ${ }^{2} \quad$ Andreas Löhne ${ }^{2} \quad$ Benjamin Weißing ${ }^{2}$
}

July 17, 2018

\begin{abstract}
The article deals with operations defined on convex polyhedra or polyhedral convex functions. Given two convex polyhedra, operations like Minkowski sum, intersection and closed convex hull of the union are considered. Basic operations for one convex polyhedron are, for example, the polar, the conical hull and the image under affine transformation. The concept of a P-representation of a convex polyhedron is introduced. It is shown that many polyhedral calculus operations can be expressed explicitly in terms of P-representations. We point out that all the relevant computational effort for polyhedral calculus consists in computing projections of convex polyhedra. In order to compute projections we use a recent result saying that multiple objective linear programming (MOLP) is equivalent to the polyhedral projection problem. Based on the MOLPsolver bensolve a polyhedral calculus toolbox for Matlab and GNU Octave is developed. Some numerical experiments are discussed.
\end{abstract}

Keywords: polyhedron, polyhedral set, polyhedral convex analysis, polyhedron computations, multiple objective linear programming, P-representation

MSC 2010 Classification: 52B55, 90C29

\section{Introduction}

Convex polyhedra and polyhedral convex functions are relevant in many disciplines of mathematics and sciences. They can be used to approximate convex sets and convex functions with the advantage of always having finite representations. This naturally leads to the need of a calculus, that is, a collection of

\footnotetext{
${ }^{1}$ This research was supported by the German Research Foundation (DFG) grant number LO-1379/7-1.

${ }^{2}$ Friedrich Schiller University Jena, Department of Mathematics, 07737 Jena, Germany, [daniel.ciripoi|andreas.loehne|benjamin.weissing]@uni-jena.de
} 
operations on one, two or even finitely many convex polyhedra or polyhedral convex functions.

We introduce a P-representation of a convex polyhedron, where the ' $\mathrm{P}$ ' stands for 'projection', and show that typical calculus operations can be expressed explicitly in terms of P-representations. It turns out that all the computational effort in polyhedral calculus consists in computing H-representations (inequality representations) and/or V-representations (representations by finitely many points and directions) from P-representations. To this end we utilize a multiple objective linear programming solver based on the fact that polyhedral projection is equivalent to multiple objective linear programming [11.

Motivated by the relevance of P-representations for polyhedral calculus, it appears to be natural to define a convex polyhedron as follows:

Definition 1. Given matrices $M \in \mathbb{R}^{q \times n}, B \in \mathbb{R}^{m \times n}$, and vectors

$$
a \in(\mathbb{R} \cup\{-\infty\})^{m}, b \in(\mathbb{R} \cup\{+\infty\})^{m}, l \in(\mathbb{R} \cup\{-\infty\})^{n}, u \in(\mathbb{R} \cup\{+\infty\})^{n},
$$

the set

$$
P=\{M x \mid a \leqslant B x \leqslant b, l \leqslant x \leqslant u\}
$$

is called a convex polyhedron ${ }^{1}$. The tuple $(M, B, a, b, l, u)$ is called $\mathrm{P}$-representation of the convex polyhedron $P$.

Thus, a polyhedral set is the $q$-dimensional set we obtain by mapping the points in $\mathbb{R}^{n}$ which are bounded by the vectors $l$ and $u$ and satisfy the given system of $2 m$ affine inequalities given by $a, b$ and $B$. We use the symbol $\varnothing$ to indicate components that do not occur. For example, $(M, \varnothing, \varnothing, \varnothing, l, u)$ represents the polyhedron $P=\{M x \mid l \leqslant x \leqslant u\}$ and $(M, B, a, \varnothing, l, \varnothing)$ represents $P=\{M x \mid a \leqslant B x, l \leqslant x\}$.

In the literature, see e.g. [13, a polyhedron is usually defined as an intersection of finitely many closed half-spaces, which refers to the special case of $M$ being the unit matrix and thus $q=n$. In this case, we have

$$
P=\{x \mid a \leqslant B x \leqslant b, l \leqslant x \leqslant u\}
$$

and the tuple $(B, a, b, l, u)$ is called $H$-representation of $P$.

A simple reformulation shows that (1) is the projection of an H-represented polyhedron

$$
Q=\left\{(x, y) \in \mathbb{R}^{n} \times \mathbb{R}^{q} \mid y=M x, a \leqslant B x \leqslant b, l \leqslant x \leqslant u\right\}
$$

onto the $y$-components, which motivates the term P-representation.

Fourier-Motzkin-Elimination, see e.g. [8], provides a tool for eliminating the $x$-components in the following reformulation of (1):

$$
P=\left\{y \in \mathbb{R}^{q} \mid \exists x \in \mathbb{R}^{n}: y=M x, a \leqslant B x \leqslant b, l \leqslant x \leqslant u\right\} .
$$

\footnotetext{
${ }^{1}$ We solely deal with convex polyhedra in this paper, thus we omit the term 'convex' in subsequent occurrences.
} 
This means that every polyhedron as defined in Definition 1 admits an H-representation, which justifies to define a polyhedron in an alternative way via a P-representation.

It should be pointed out that in contrast to 22 the H-representation of a polyhedron is usually defined as $P=\{x \mid B x \leqslant b\}$ in the literature. The seemingly redundant form we use in (2), where we distinguish between constraints and variable bounds and where double bounds are employed, resembles the input format of bensolve tools. Using this more explicit formulation, users can specify polyhedra directly as they appear in their respective applications without the need to reformulate the defining system in the form $B x \leqslant b$.

According to the Minkowski-Weyl theorem, every polyhedron admits a representation in terms of points and directions: Consider a polyhedron $P \subseteq \mathbb{R}^{q}$ and let matrices $V \in \mathbb{R}^{q \times r}(r \geqslant 1), D \in \mathbb{R}^{q \times s}(s \geqslant 0)$ and $L \in \mathbb{R}^{q \times t}(t \geqslant 0)$ be given, where we write $D=\varnothing$ and $L=\varnothing$ if $s=0$ and $t=0$, respectively. The vectors $v^{1}, \ldots, v^{r}, d^{1}, \ldots, d^{s}$ and $l^{1}, \ldots, l^{s}$ shall denote the columns of $V$, $D$ and $L$, respectively. If

$$
P=\operatorname{conv}\left\{v^{1}, \ldots, v^{r}\right\}+\text { cone }\left\{d^{1}, \ldots, d^{s}\right\}+\operatorname{span}\left\{l^{1}, \ldots, l^{s}\right\}
$$

holds, where we set cone $\varnothing=\{0\}$ and $\operatorname{span} \varnothing=\{0\}$, then $(V, D, L)$ is called $V$-representation of $P$.

Given a V-representation $(V, D, L)$ of a polyhedron, it is evident that

$$
\left((V, D, L),\left(1_{(r)}^{\top}, 0_{(s+t)}^{\top}\right), 1,1,\left(\begin{array}{c}
0_{(r+s)} \\
-\infty_{(t)}
\end{array}\right), \infty_{(r+s+t)}\right)
$$

is a P-representation of $P$, where $a_{(n)}$ stands for an $n$-dimensional column vector all the $n$ components of which equal to $a$.

The problem of computing a V-representation for a polyhedron given in $\mathrm{H}$ representation is called the vertex enumeration problem. The reverse problem is called facet enumeration problem and can be interpreted as vertex enumeration problem under polarity. Since an H-representation is a special case of a P-representation, vertex enumeration can be seen as special case of the polyhedral projection problem [14, which is, roughly speaking, the problem to compute a V-representation from a P-representation. Analogously, the dual polyhedral projection problem as introduced in [14] covers facet enumeration.

An idea related to the one presented here is used in 12 for treating combinatorial optimization problems. There, the authors make use of the fact that a high dimensional polyhedron may have a simple structure, while some low dimensional projection may become quite complex.

Another polyhedral calculus toolbox, which also covers non-convex polyhedra, is MPT3 [6]. In Section 5 we compare MPT3 to our approach.

This article is organized as follows. In Section 2 we present the results about calculus of polyhedral sets in terms of P-representations. In Section 3 , we extend the results to polyhedral functions. Section 4 discusses how a MOLP solver can be utilized to compute $\mathrm{H}$-representations and V-representations from P-representations. Section 5 introduces the polyhedral calculus software bensolve tools by discussing some numerical experiments. 


\section{Polyhedral set calculus via P-representations}

The notion of P-representation as introduced in Definition 1 allows the explicit expression of several polyhedral calculus operations. We start this section by listing some results that can be proven easily by employing the corresponding definitions.

Proposition 2 (Minkowski sum). Let the two polyhedra $A^{1} \subseteq \mathbb{R}^{q}$ and $A^{2} \subseteq \mathbb{R}^{q}$ with $P$-representations $\left(M^{1}, B^{1}, a^{1}, b^{1}, l^{1}, u^{1}\right)$ and $\left(M^{2}, B^{2}, a^{2}, b^{2}, l^{2}, u^{2}\right)$, respectively, be given. Then the sum

$$
A^{1}+A^{2}=\left\{y \in \mathbb{R}^{q} \mid \exists y^{1} \in A^{1}, y^{2} \in A^{2}: y=y^{1}+y^{2}\right\}
$$

has the P-representation

$$
\left(\left(\begin{array}{ll}
M^{1} & M^{2}
\end{array}\right),\left(\begin{array}{cc}
B^{1} & 0 \\
0 & B^{2}
\end{array}\right),\left(\begin{array}{l}
a^{1} \\
a^{2}
\end{array}\right),\left(\begin{array}{l}
b^{1} \\
b^{2}
\end{array}\right),\left(\begin{array}{l}
l^{1} \\
l^{2}
\end{array}\right),\left(\begin{array}{l}
u^{1} \\
u^{2}
\end{array}\right)\right) .
$$

Proposition 3 (intersection). Let the polyhedra $A^{1} \subseteq \mathbb{R}^{q}$ and $A^{2} \subseteq \mathbb{R}^{q}$ with $P$ representations $\left(M^{1}, B^{1}, a^{1}, b^{1}, l^{1}, u^{1}\right)$ and $\left(M^{2}, B^{2}, a^{2}, b^{2}, l^{2}, u^{2}\right)$, respectively, be given. Then the intersection $A^{1} \cap A^{2} \subseteq \mathbb{R}^{q}$ is a polyhedron with P-representation

$$
\left.\left(\begin{array}{ll}
M^{1} & 0
\end{array}\right),\left(\begin{array}{cc}
B^{1} & 0 \\
0 & B^{2} \\
M^{1} & -M^{2}
\end{array}\right),\left(\begin{array}{l}
a^{1} \\
a^{2} \\
0
\end{array}\right),\left(\begin{array}{l}
b^{1} \\
b^{2} \\
0
\end{array}\right),\left(\begin{array}{l}
l^{1} \\
l^{2}
\end{array}\right),\left(\begin{array}{l}
u^{1} \\
u^{2}
\end{array}\right)\right) .
$$

Proposition 4 (Cartesian product). Let the polyhedra $A^{1} \subseteq \mathbb{R}^{p}$ and $A^{2} \subseteq \mathbb{R}^{q}$ with P-representations $\left(M^{1}, B^{1}, a^{1}, b^{1}, l^{1}, u^{1}\right)$ and $\left(M^{2}, B^{2}, a^{2}, b^{2}, l^{2}, u^{2}\right)$, respectively, be given. Then the Cartesian product $A^{1} \times A^{2} \subseteq \mathbb{R}^{p+q}$ is a polyhedron with P-representation

$$
\left(\left(\begin{array}{cc}
M^{1} & 0 \\
0 & M^{2}
\end{array}\right),\left(\begin{array}{cc}
B^{1} & 0 \\
0 & B^{2}
\end{array}\right),\left(\begin{array}{l}
a^{1} \\
a^{2}
\end{array}\right),\left(\begin{array}{l}
b^{1} \\
b^{2}
\end{array}\right),\left(\begin{array}{l}
l^{1} \\
l^{2}
\end{array}\right),\left(\begin{array}{l}
u^{1} \\
u^{2}
\end{array}\right)\right) .
$$

Proposition 5 (recession cone). Consider the polyhedron $A \subseteq \mathbb{R}^{q}$ with P-representation $(M, B, a, b, l, u)$. The recession cone of $A$,

$$
\operatorname{recc} A=\left\{y \in \mathbb{R}^{q} \mid \forall x \in A, \forall t \geqslant 0: x+t y \in A\right\}
$$

has the P-representation $(M, B, 0 \cdot a, 0 \cdot b, 0 \cdot l, 0 \cdot u)$, where we set $0 \cdot \pm \infty:= \pm \infty$.

We close the first part of this section by an example for the Minkowski sum.

Example 6. Let $A^{1} \subseteq \mathbb{R}^{q}$ be the unit ball of the 1-norm,

$$
A^{1}=\left\{y \in \mathbb{R}^{q}\left|\sum_{i=1}^{q}\right| y_{i} \mid \leqslant 1\right\},
$$

and let

$$
A^{2}=\left\{y \in \mathbb{R}^{q} \mid-1 \leqslant y \leqslant 1\right\}
$$



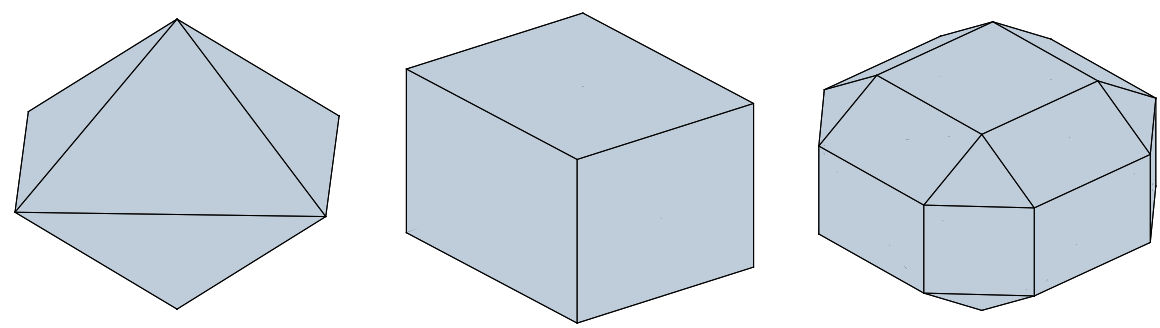

Figure 1: From left to right: 3-dimensional unit ball of 1-norm, 3-dimensional unit ball of $\infty$-norm, Minkowski sum of the unit balls.

be the unit ball of the $\infty$-norm in $\mathbb{R}^{q}$. Both norms are polyhedral, thus $A^{1}$ and $A^{2}$ are polyhedra. Since the set $A^{1}$ is the convex hull of the unit vectors and their negatives, it can be expressed as

$$
\begin{aligned}
A^{1} & =\left\{y \in \mathbb{R}^{q}\left|\sum_{i=1}^{q}\right| y_{i} \mid \leqslant 1\right\} \\
& =\left\{x-z \mid x, z \in \mathbb{R}_{+}^{q}, \sum_{i=1}^{q} x_{i}+z_{i}=1\right\} .
\end{aligned}
$$

This produces a P-representation which is given by

$$
\left(\left(I_{q} \quad-I_{q}\right), 1_{(2 q)}^{\top}, 1,1,0_{(2 q)}, \infty{ }_{(2 q)}\right),
$$

where $I_{q}$ denotes the $q \times q$ unit matrix. Polyhedron $A^{2}$ admits the P-representation

$$
\left(I_{q}, \varnothing, \varnothing, \varnothing,-1_{(q)}, 1_{(q)}\right) .
$$

Therefore, by Proposition 2 we obtain the P-representation

$$
\left(\left(\begin{array}{lll}
I_{q} & -I_{q} & I_{q}
\end{array}\right),\left(1_{(2 q)}^{\top}, 0_{(q)}^{\top}\right), 1,1,\left(\begin{array}{c}
0_{(2 q)} \\
-1_{(q)}
\end{array}\right),\left(\begin{array}{c}
\infty_{(2 q)} \\
1_{(q)}
\end{array}\right)\right)
$$

for the polyhedron $A^{1}+A^{2}$. We illustrate the sets $A^{1}, A^{2}$ and their Minkowski sum $A^{1}+A^{2}$ for $q=3$ in Figure 1 .

Other polyhedral calculus operations like computing the polar of a nonempty polyhedron or computing the closed convex hull of the union of finitely many polyhedra can be obtained by utilizing a variant of the Farkas lemma like Motzkin's transposition theorem. Also in these cases, the resulting P-representations only require transposition and rearrangement of the given data.

Theorem 7 (Motzkin's transposition theorem). The linear system

$$
B_{1} x<b_{1}, \quad B_{2} x \leqslant b_{2}, \quad B_{3} x=b_{3}
$$


has no solution if and only if there exist $z_{0} \in \mathbb{R}$ and vectors $z_{1}, z_{2}, z_{3}$ such that

$$
\begin{aligned}
z_{1}^{\top} B_{1}+z_{2}^{\top} B_{2}+z_{3}^{\top} B_{3} & =0, \\
z_{0}+z_{1}^{\top} b_{1}+z_{2}^{\top} b_{2}+z_{3}^{\top} b_{3} & =0, \\
\left(\begin{array}{l}
z_{0} \\
z_{1} \\
z_{2}
\end{array}\right) \geqslant 0, \quad\left(\begin{array}{l}
z_{0} \\
z_{1}
\end{array}\right) & \neq 0 .
\end{aligned}
$$

Proof. See, for instance, [4, Theorem 3.17 / Section 3.3].

Proposition 8. The polar

$$
A^{\circ}=\left\{y \in \mathbb{R}^{q} \mid \forall v \in A: y^{\top} v \leqslant 1\right\} .
$$

of a nonempty polyhedron $A \subseteq \mathbb{R}^{q}$ with P-representation ( $\left.M, B, a, b, l, u\right)$, where $B \in \mathbb{R}^{m \times n}$, has the P-representation

$$
\left(\left(0_{(q \times k)} I_{q}\right), B^{\prime},\left(\begin{array}{c}
0_{(n)} \\
-\infty
\end{array}\right),\left(\begin{array}{c}
0_{(n)} \\
1
\end{array}\right),\left(\begin{array}{c}
0_{(k)} \\
-\infty_{(q)}
\end{array}\right), \infty_{(k+q)}\right),
$$

where $B^{\prime} \in \mathbb{R}^{(n+1) \times k}$ results from

$$
\left(\begin{array}{ccccc}
B^{\top} & -B^{\top} & I_{n} & -I_{n} & -M^{\top} \\
b^{\top} & -a^{\top} & u^{\top} & -l^{\top} & 0_{(q)}^{\top}
\end{array}\right)
$$

by deleting all columns with infinite entries.

Proof. We have $y \in A^{\circ}$ if and only if

$$
v \in A \Rightarrow y^{\top} v \leqslant 1
$$

This is equivalent to the following system being inconsistent:

$$
v=M x, a \leqslant B x \leqslant b, l \leqslant x \leqslant u, y^{\top} v>1 .
$$

By Theorem 7, (6) has no solution if and only if

$$
\begin{aligned}
& \left(\begin{array}{c}
0_{(n)} \\
-y
\end{array}\right) z_{1}+\left(\begin{array}{cccc}
B^{\top} & -B^{\top} & I_{n} & -I_{n} \\
0 & 0 & 0 & 0
\end{array}\right) z_{2}+\left(\begin{array}{c}
-M^{\top} \\
I_{q}
\end{array}\right) z_{3}=0 \\
& z_{0}-z_{1}+\left(\begin{array}{llll}
b^{\top} & -a^{\top} \quad u^{\top} & -l^{\top}
\end{array}\right) z_{2}+0_{(q)}^{\top} z_{3}=0 \\
& \left(\begin{array}{l}
z_{0} \\
z_{1} \\
z_{2}
\end{array}\right) \geqslant 0, \quad\left(\begin{array}{l}
z_{0} \\
z_{1}
\end{array}\right) \neq 0
\end{aligned}
$$

has a solution, where we assume that the $\pm \infty$-components of the vector before $z_{2}$ and the corresponding columns of the matrix before $z_{2}$ have been deleted. 
Consider the case where $z_{1} \neq 0$. Then, without loss of generality, we can assume $z_{1}=1$ and by obtaining $y=z_{3}$ from the second row of the first equation in system (7) respective system can be expressed as

$$
\begin{aligned}
\left(\begin{array}{c}
0_{(n)} \\
-\infty
\end{array}\right) \leqslant\left(\begin{array}{ccccc}
B^{\top} & -B^{\top} & I_{n} & -I_{n} & -M^{\top} \\
b^{\top} & -a^{\top} & u^{\top} & -l^{\top} & 0_{(q)}^{\top}
\end{array}\right)\left(\begin{array}{c}
z_{2} \\
y
\end{array}\right) \leqslant\left(\begin{array}{c}
0_{(n)} \\
1
\end{array}\right) \\
z_{2} \geqslant 0,
\end{aligned}
$$

which yields the P-representation claimed for $A^{\circ}$.

Finally we show that the case $z_{1}=0$ cannot occur. Otherwise, we get $z_{0}>0$ and $z_{3}=0$. Hence, system (7) turns into

$$
\begin{aligned}
& \left(\begin{array}{llll}
B^{\top} & -B^{\top} & I_{n} & -I_{n}
\end{array}\right) z_{2}=0 \\
& \left(\begin{array}{llll}
b^{\top} & -a^{\top} & u^{\top} & -l^{\top}
\end{array}\right) z_{2}<0 \\
& z_{2} \geqslant 0 \text {. }
\end{aligned}
$$

As $A$ is assumed to be nonempty, we can choose an element $\bar{x}$ such that

$$
\bar{x}^{\top}\left(\begin{array}{llll}
B^{\top} & -B^{\top} & I_{n} & -I_{n}
\end{array}\right) \leqslant\left(\begin{array}{llll}
b^{\top} & -a^{\top} & u^{\top} & -l^{\top}
\end{array}\right) .
$$

This leads to the contradiction

$$
0=\bar{x}^{\top}\left(\begin{array}{llll}
B^{\top} & -B^{\top} & I_{n} & -I_{n}
\end{array}\right) z_{2} \leqslant\left(\begin{array}{llll}
b^{\top} & -a^{\top} & u^{\top} & -l^{\top}
\end{array}\right) z_{2}<0,
$$

which completes the proof.

Proposition 9. The polar cone

$$
A^{*}=\left\{y \in \mathbb{R}^{q} \mid \forall x \in A: y^{\top} x \leqslant 0\right\}
$$

of a nonempty polyhedron $A \subseteq \mathbb{R}^{q}$ with P-representation $(M, B, a, b, l, u)$ has the P-representation

$$
\left(\left(0_{(q \times k)} I_{q}\right), B^{\prime},\left(\begin{array}{c}
0_{(n)} \\
-\infty
\end{array}\right), 0_{(n+1)},\left(\begin{array}{c}
0_{(k)} \\
-\infty_{(q)}
\end{array}\right), \infty_{(k+q)}\right),
$$

where $B^{\prime}$ is the same matrix as in Proposition 8.

Proof. Similar to the proof of Proposition 8

We now recall some well-known results on polyhedra which allow us to express further polyhedral calculus operations in terms of P-representations. Let $A^{\circ \circ}=\left(A^{\circ}\right)^{\circ}$ and $A^{* *}=\left(A^{*}\right)^{*}$ denote the bipolar and the bipolar cone of a polyhedron $A$.

Proposition 10 (see e.g. [13). For nonempty polyhedra $A, A_{1}, A_{2} \subseteq \mathbb{R}^{q}$ and nonempty polyhedral cones $C, C_{1}, C_{2} \subseteq \mathbb{R}^{q}$ one has

(i) $A^{\circ \circ}=\operatorname{cl} \operatorname{conv}\{A \cup\{0\}\}$, 
(ii) If $0 \in A$, then $A^{\circ \circ}=A$,

(iii) $C^{* *}=C$,

(iv) cl cone $A=A^{* *}$,

(v) If $0 \in A_{1} \cup A_{2}$, then $\operatorname{cl} \operatorname{conv}\left(A_{1} \cup A_{2}\right)=\left(\left(A_{1}\right)^{\circ} \cap\left(A_{2}\right)^{\circ}\right)^{\circ}$,

(vi) $C_{1}+C_{2}=\left(\left(C_{1}\right)^{\circ} \cap\left(C_{2}\right)^{\circ}\right)^{\circ}$.

Note that the closure operation cannot be omitted in the polyhedral case (take for instance $A=\left\{x \in \mathbb{R}^{2} \mid x_{2}=1\right\}$ in (i)).

As a consequence of the preceding proposition we are able to derive a $\mathrm{P}$ representation of the closed conic hull of a polyhedron by applying the polar cone operation twice, see (iv). Furthermore, we obtain a P-representation of the closed convex hull of the union of two polyhedra ((v) and translation). The normal cone of a polyhedron $A \subseteq \mathbb{R}^{q}$ at a point $\bar{x} \in A$ is defined as the set

$$
\mathrm{N}_{A}\left(x_{0}\right)=\left\{y \in \mathbb{R}^{q} \mid \forall x \in A: y^{\top}(x-\bar{x}) \leqslant 0\right\} .
$$

It is known (see e.g. 13]) that,

$$
\mathrm{N}_{A}(\bar{x})=(\operatorname{cone}(A-\{\bar{x}\}))^{\circ} .
$$

Thus, a P-representation of $\mathrm{N}_{A}(\bar{x})$ is obtained by combining some of the previous results.

\section{Calculus of polyhedral convex functions}

A function $f: \mathbb{R}^{n} \rightarrow \mathbb{R} \cup\{+\infty\}$ is called polyhedral if its epigraph

$$
\text { epi } f=\left\{(x, r) \in \mathbb{R}^{n} \times \mathbb{R} \mid f(x) \leqslant r\right\}
$$

is a polyhedron. Since all polyhedra in this article are convex, polyhedral functions are convex, too. The domain of $f$ is defined as

$$
\operatorname{dom} f=\left\{x \in \mathbb{R}^{n} \mid f(x)<+\infty\right\} .
$$

A polyhedral function can be represented by a P-representation of its epigraph. Well-known results from Convex Analysis provide the relationship between function operations and corresponding epigraph operations. Thus, using our calculus for polyhedral sets applied to the epigraphs, we can easily derive calculus operations for polyhedral functions. If a polyhedral function $f$ is represented by a P-representation $(M, B, a, b, l, u)$ of its epigraph, then computing a function value $f(x)$ for some $x \in \mathbb{R}^{n}$ requires to solve the linear program

$$
\min _{r, z} r \text { s.t. }\left(\begin{array}{l}
x \\
r
\end{array}\right)=M z, a \leqslant B z \leqslant b, l \leqslant z \leqslant u .
$$

The next four statements combined with the results of Section 2 provide some first calculus operations for polyhedral functions. Proofs can be found in Convex Analysis books such as [13. 
Proposition 11. Let $f_{1}, \ldots, f_{k}: \mathbb{R}^{n} \rightarrow \mathbb{R} \cup\{+\infty\}$ be polyhedral functions. The epigraph of the pointwise maximum function $\max \left(f_{1}, \ldots, f_{k}\right)$ is

$$
\operatorname{epi} \max \left(f_{1}, \ldots, f_{k}\right)=\bigcap_{i=1}^{k} \operatorname{epi} f_{i} .
$$

The lower closed convex envelope $\operatorname{lenv}\left(f_{1}, \ldots, f_{k}\right)$ of given polyhedral functions $f_{1}, \ldots, f_{k}: \mathbb{R}^{n} \rightarrow \mathbb{R} \cup\{+\infty\}$ is defined as the largest closed convex function from $\mathbb{R}^{n}$ to $\mathbb{R} \cup\{+\infty\}$ majorized by all given functions.

Proposition 12. Let $f_{1}, \ldots, f_{k}: \mathbb{R}^{n} \rightarrow \mathbb{R} \cup\{+\infty\}$ be polyhedral functions. The epigraph of the lower closed convex envelope function lenv $\left(f_{1}, \ldots, f_{k}\right)$ is

$$
\text { epi lenv }\left(f_{1}, \ldots, f_{k}\right)=\operatorname{clconv}\left(\bigcup_{i=1}^{k} \operatorname{epi} f_{i}\right) \text {. }
$$

The infimal convolution of polyhedral functions $f_{1}, \ldots, f_{k}: \mathbb{R}^{n} \rightarrow \mathbb{R} \cup\{+\infty\}$ is defined as

$$
\left(f_{1} \square \ldots \square f_{k}\right)(x)=\inf \left\{f_{1}\left(x^{1}\right)+\cdots+f_{k}\left(x^{k}\right) \mid x^{1}+\cdots+x^{k}=x\right\} .
$$

Proposition 13. Let $f_{1}, \ldots, f_{k}: \mathbb{R}^{n} \rightarrow \mathbb{R} \cup\{+\infty\}$ be polyhedral functions. Then we have

$$
\operatorname{epi}\left(f_{1} \square \ldots \square f_{k}\right)=\operatorname{epi} f_{1}+\cdots+\operatorname{epi} f_{k} .
$$

Proposition 14. Let $f_{1}, \ldots, f_{k}: \mathbb{R}^{n} \rightarrow \mathbb{R} \cup\{+\infty\}$ be polyhedral functions. For the pointwise sum function $f_{1}+\ldots+f_{k}$, one has

$\operatorname{epi}\left(f_{1}+\ldots+f_{k}\right)=\left\{(x, r) \in \mathbb{R}^{n} \times \mathbb{R} \mid \sum_{i=1}^{k} r_{i}=r,\left(x, r_{i}\right) \in \operatorname{epi} f_{i}, i=1, \ldots, k\right\}$.

The conjugate $f^{*}: \mathbb{R}^{n} \rightarrow \mathbb{R} \cup\{+\infty\}$ of a polyhedral convex function $f:$ $\mathbb{R}^{n} \rightarrow \mathbb{R} \cup\{+\infty\}$ with $\operatorname{dom} f \neq \varnothing$ is defined as

$$
f^{*}\left(x^{*}\right)=\sup _{x \in \operatorname{dom} f}\left\{x^{\top} x^{*}-f(x)\right\} .
$$

The following result tells us that a P-representation of the the epigraph of $f^{*}$ can be obtained from a P-representation of the epigraph of $f$ by polyhedral calculus operations as discussed in Section 2

Proposition 15. Let $f: \mathbb{R}^{n} \rightarrow \mathbb{R} \cup\{+\infty\}$ be a polyhedral function with $\operatorname{dom} f \neq$ $\varnothing$. Then,

$$
\text { epi } f^{*}=\left\{\left(x^{*}, r^{*}\right) \mid\left(x^{*},-1, r^{*}\right) \in K(f)^{*}\right\},
$$

where $K(f)^{*}$ is the polar cone of the polyhedron

$$
K(f)=\{(x, r,-1) \mid(x, r) \in \text { epi } f\} .
$$


Proof. The epigraph of $f^{*}$ is the set

$$
\begin{aligned}
\text { epi } f^{*} & =\left\{\left(x^{*}, r^{*}\right) \mid f^{*}\left(x^{*}\right) \leqslant r^{*}\right\} \\
& =\left\{\left(x^{*}, r^{*}\right) \mid \sup _{x \in \operatorname{dom} f}\left\{x^{\top} x^{*}-f(x)\right\} \leqslant r^{*}\right\} \\
& =\left\{\left(x^{*}, r^{*}\right) \mid \forall x \in \operatorname{dom} f: x^{\top} x^{*}-f(x) \leqslant r^{*}\right\} \\
& =\left\{\left(x^{*}, r^{*}\right) \mid \forall(x, r) \in \operatorname{epi} f: x^{\top} x^{*}-r \leqslant r^{*}\right\} \\
& =\left\{\left(x^{*}, r^{*}\right) \mid \forall(x, r,-1) \in K(f): x^{\boldsymbol{\top}} x^{*}+r(-1)+(-1) r^{*} \leqslant 0\right\} \\
& =\left\{\left(x^{*}, r^{*}\right) \mid\left(x^{*},-1, r^{*}\right) \in K(f)^{*}\right\},
\end{aligned}
$$

which completes the proof.

Further operations for polyhedral functions can be obtained in a similar manner. For instance, the ability to compute the normal cone of a P-represented epigraph of a polyhedral function $f$ can be used to compute a P-representation of the (convex) subdifferential of $f$ at a point $x \in \operatorname{dom} f$ by using the well-known formula

$$
\partial f(x)=\left\{y \in \mathbb{R}^{n} \mid\left(\begin{array}{c}
y \\
-1
\end{array}\right) \in \mathrm{N}_{\text {epi } f}\left(\begin{array}{c}
x \\
f(x)
\end{array}\right)\right\} .
$$

\section{Computing projections via MOLP}

In this section we briefly outline how to compute the V-representation and $\mathrm{H}$ representation of a polyhedron given in P-representation. For further details concerning the theoretical background the reader is referred to [11, 14. The main idea is to solve a multiple objective linear program (MOLP) connected to the projection problem. We can then derive the V-representation and H-representation of the projected polyhedron from the primal and dual solution of the corresponding MOLP.

In multiple objective linear programming, the so-called upper image plays an important role. The upper image of

$$
\min M x \quad \text { s.t. } \quad a \leqslant B x \leqslant b, l \leqslant x \leqslant u
$$

is the polyhedron

$$
\mathcal{P}=\left\{y \in \mathbb{R}^{q} \mid \exists x \in \mathbb{R}^{n}: y \geqslant M x, a \leqslant B x \leqslant b, l \leqslant x \leqslant u\right\} .
$$

Algorithms for (MOLP) like Benson's algorithm [1, 5] compute both a V-representation and an H-representation of $\mathcal{P}$. A solution to (MOLP) as introduced in [9] is closely related to a V-representation of $\mathcal{P}$, whereas a solution of the dual problem in the sense of [7] refers in the same manner to an H-representation of $\mathcal{P}$.

The main idea of computing a V-representation and an H-representation from a given P-representation $(M, B, a, b, l, u)$ of a polyhedron $A \subseteq \mathbb{R}^{q}$ is to 
consider the problem

$$
\min \left(\begin{array}{c}
M \\
-1_{(q)}^{\top} M
\end{array}\right) x \quad \text { s.t. } \quad a \leqslant B x \leqslant b, l \leqslant x \leqslant u
$$

with upper image

$\mathcal{M}=\left\{(y, r) \in \mathbb{R}^{q} \times \mathbb{R} \mid \exists x \in \mathbb{R}^{n}: y \geqslant M x, r \geqslant-1_{(q)}^{\top} M x, a \leqslant B x \leqslant b, l \leqslant x \leqslant u\right\}$.

It is easily seen that the polyhedron $A$ can be expressed by $\mathcal{M}$ as

$$
\begin{aligned}
A & =\left\{y \in \mathbb{R}^{q} \mid \exists x \in \mathbb{R}^{n}: y=M x, a \leqslant B x \leqslant b, l \leqslant x \leqslant u\right\} \\
& =\left\{y \in \mathbb{R}^{q} \mid\left(\begin{array}{c}
y \\
-1_{(q)}^{\top} y
\end{array}\right) \in \mathcal{M}\right\} .
\end{aligned}
$$

From the V-representation and the H-representation of $\mathcal{M}$, which are obtained by solving ( $\overline{\text { MOLP' }}$, one can compute a V-representation and an $\mathrm{H}$ representation of the polyhedron $A$. Considering (8) and [11, Theorem 3], a V-representation of $A$ is obtained from a V-representation of $\mathcal{M}$ by deleting

directions $d$ with $1_{(q+1)}^{\top} d \neq 0$ and by omitting the $(q+1)$-th components of all remaining vectors.

An H-representation of $A$ is easily generated by the H-representation of $\mathcal{M}$ employing (8). If the tuple $\left(B^{1}, a^{1}, b^{1}, l^{1}, u^{1}\right)$ is an H-representation of $\mathcal{M}$ comprising $2 m$ affine inequalities, i.e. $B^{1} \in \mathbb{R}^{m \times(q+1)}$, then, by replacing the last component of the unknown by the negative sum of the first $q$ components we obtain the H-representation of $A$ satisfying the system of inequalities given by

$$
\left(\begin{array}{c}
a^{1} \\
l_{q+1}^{1}
\end{array}\right) \leqslant\left(\begin{array}{ccc}
B_{11}^{1}-B_{1(q+1)}^{1} & \ldots & B_{1 q}^{1}-B_{1(q+1)}^{1} \\
\vdots & \ddots & \vdots \\
B_{m 1}^{1}-B_{m(q+1)}^{1} & \ldots & B_{m q}^{1}-B_{m(q+1)}^{1} \\
-1 & \ldots & -1
\end{array}\right) y \leqslant\left(\begin{array}{c}
b^{1} \\
u_{q+1}^{1}
\end{array}\right)
$$

and

$$
\left(\begin{array}{c}
l_{1}^{1} \\
\vdots \\
l_{q}^{1}
\end{array}\right) \leqslant y \leqslant\left(\begin{array}{c}
u_{1}^{1} \\
\vdots \\
u_{q}^{1}
\end{array}\right) .
$$

\section{$5 \quad$ Numerical experiments}

The goal of this section is twofold. First we consider an example from locational analysis in order to demonstrate how polyhedral calculus can be used for modeling polyhedral convex optimization problems. Secondly, we compare our implementation bensolve tools [2] with another polyhedral calculus software by projecting high dimensional polyhedra. 
Bensolve tools is a free and open source software for GNU Octave and Matlab. It utilizes the VLP solver bensolve [10, which is written in C programming language. The recent version of bensolve tools 2 has the following features:

- calculus of convex polyhedra,

- calculus of polyhedral convex function,

- solver for polyhedral convex programs (via LP reformulation),

- solver for vector linear programs and multiple objective linear programs (bensolve interface),

- solver for quasi-convace global optimization problems, see [3] for details.

\subsection{Polyhedral location problems}

Let a finite number of points $a^{1}, \ldots, a^{m} \in \mathbb{R}^{n}$ be given and let $d: \mathbb{R}^{n} \times \mathbb{R}^{n} \rightarrow \mathbb{R}$ be a metric. We consider the location optimization problem

$$
\min _{x \in \mathbb{R}^{n}} \sum_{i=1}^{m} d\left(x, a^{i}\right)
$$

Let $G_{i} \subseteq \mathbb{R}^{n}$ be bounded polyhedra with $0 \in \operatorname{int} G_{i}$ and let $g_{i}: \mathbb{R}^{n} \rightarrow \mathbb{R}$ be the corresponding gauge function, which can be defined by

$$
\text { epi } g_{i}=\text { cone }\left(G_{i} \times\{1\}\right) \text {. }
$$

Then $g_{i}$ is a polyhedral convex function and $d(z, y)=g_{i}(z-y)$ is a metric.

The distance from $x$ to $a^{i}$ can be expressed by a function $f_{i}: \mathbb{R}^{n} \rightarrow \mathbb{R}$, $f_{i}(x)=g_{i}\left(x-a^{i}\right)$. Its epigraph is

$$
\text { epi } f_{i}=\text { epi } g_{i}+\left(\begin{array}{c}
a^{i} \\
0
\end{array}\right) \text {. }
$$

The location problem (9) can be written as

$$
\min _{x \in \mathbb{R}^{n}} \sum_{i=1}^{m} f_{i}(x) .
$$

Now it is evident that a P-representation $(M, B, a, b, l, u)$ of the objective function $f: \mathbb{R}^{n} \rightarrow \mathbb{R}, f(x)=\sum_{i=1}^{m} f_{i}(x)$ can be obtained by the polyhedral calculus operations discussed above. Thus, if $\left(x^{*}, r^{*}, z^{*}\right)$ is a solution of the linear program

$$
\min _{x, r, z} r \text { s.t. }\left(\begin{array}{l}
x \\
r
\end{array}\right)=M z, a \leqslant B z \leqslant b, l \leqslant z \leqslant u,
$$

then $x^{*}$ is an optimal solution of 10 . 
Example 16. Let $B_{1}$ be the unit ball of the 1-norm and $B_{\infty}$ be the unit ball of the $\infty$-norm. For all $i$, we set $G_{i}=B_{1}+B_{\infty}$, see Figure 1 in Section 2 for the case $n=3$. The points $a^{i}$ are generated randomly on a grid. The resulting problem instances are solved by bensolve tools. To this end, the objective function $f$ is composed from the data and then the integrated solver for polyhedral convex programs is used. The set of all solutions of (10) can be obtained in different ways, here it is obtained by computing the subdifferential of the conjugate of $f$ at 0 using the corresponding bensolve tools commands. The results are illustrated in Figures 2 and 3.
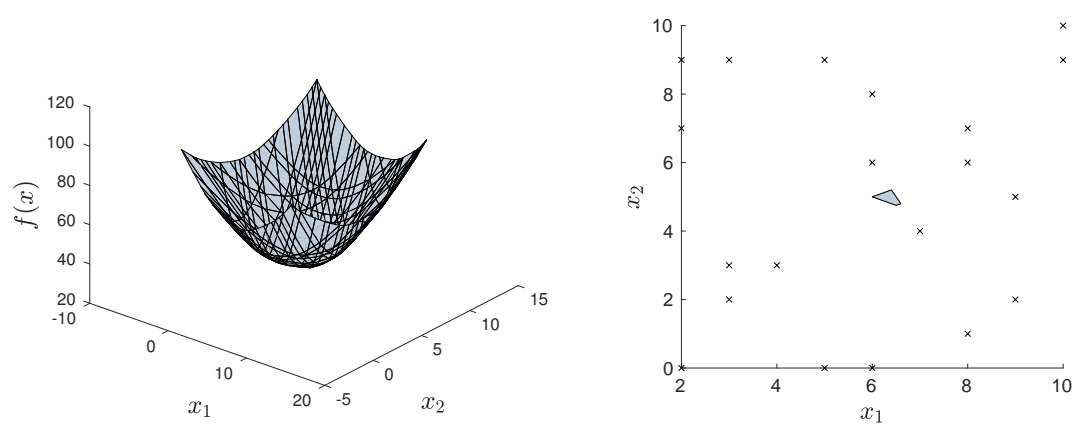

Figure 2: Left: the epigraph of $f$; Right: the given points $a^{i}$ and the set of all optimal solutions.
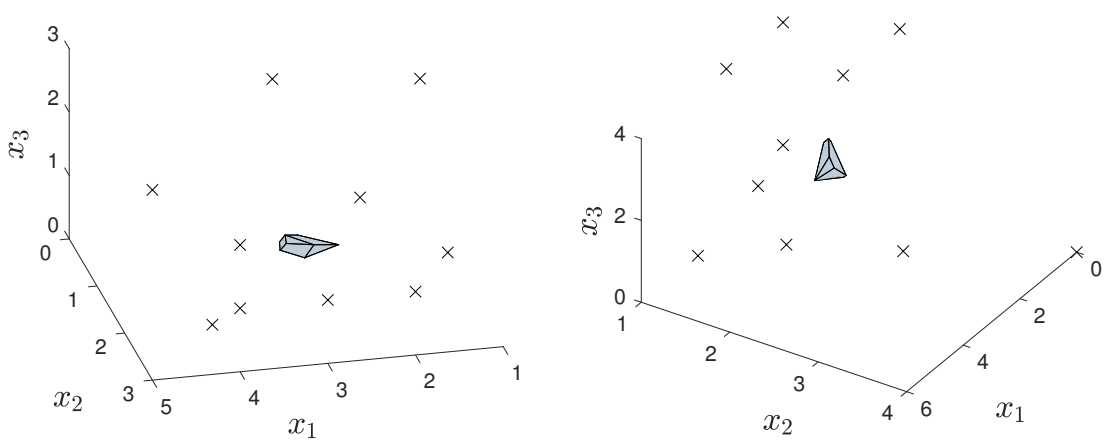

Figure 3: The given points $a^{i}$ and the set of all optimal solutions for two instances of the case $n=3$.

\subsection{Projection of high dimensional polyhedra}

The preceding results show that polyhedral projection is a key tool for polyhedral calculus. Thus, any projection algorithm computing a V-representation 
and an H-representation can be employed to carry out polyhedral calculus operations. While bensolve tools uses the projection algorithm from Section 4 which works in the image space $\mathbb{R}^{q}$, MPT3 uses a projection technique which works in the variable space $\mathbb{R}^{n}$. The P-represented polyhedra arising in the polyhedral calculus framework typically encode a projection from a high-dimensional variable space into a low-dimensional image space, i.e. $q \ll n$. Thus, with projection algorithms which work in this low-dimensional image space, one will achieve better results than using algorithms which work in the high-dimensional variable space $\mathbb{R}^{n}$. In order to support this claim, we compare the performances of bensolve tools and the multi-parametric toolbox MPT3 [6] for randomly generated projection problems in Example 17.

It should be noted that MPT3 has several features such as calculus operations for nonconvex polyhedra, which are not covered by the recent version of bensolve tools. Moreover, MPT3 provides different projection algorithms, which might be favorable for other examples.

Example 17. Consider an H-representation $(B, a, \varnothing, \varnothing, \varnothing)$ of a polyhedron $P=\left\{x \in \mathbb{R}^{n} \mid B x \geqslant a\right\}$ which consists of $m=3 n$ constraints. Let the matrix $B \in \mathbb{R}^{m \times n}$ consist of uniformly distributed (pseudo-)random numbers out of the interval $[-1 / 2,1 / 2]$. We determine the vector $a \in \mathbb{R}^{m}$ such that the $n$ dimensional simplex $S$ is contained in $P$. This is achieved by setting $a_{i}$ to the minimum of the $i$-th row of the matrix $\left(B, 0_{(n)}\right)$. We project $P$ onto its first $q$ components. Then, the $\mathrm{H}$ - as well as the V-representation of the resulting polyhedron are calculated. Figure 4 shows numerical results of different instances of this problem computed with bensolve tools and MPT3.
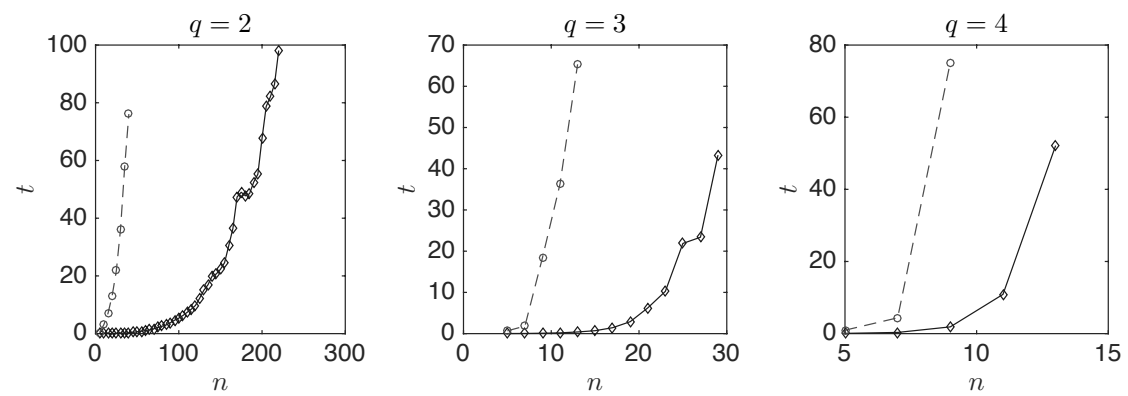

Figure 4: Comparison of bensolve tools (solid line) and MPT3 [6] (dashed line). The average CPU time $t$ in seconds of 10 random instances of Example 17 is displayed. The CPU time per instance is limited to 100 seconds. The projection method in MPT3 is run with option 'mplp' to achieve the best performance. The standard options of bensolve tools are used. 


\section{Conclusions}

In this article, we show how P-representations can be used to perform polyhedral calculus in an efficient and straight-forward manner. We demonstrate how the results for polyhedral calculus can be applied to calculus for polyhedral convex functions. Moreover, we provide methods and the software bensolve tools for polyhedral calculus based on the MOLP solver bensolve.

\section{References}

[1] H. P. Benson. An outer approximation algorithm for generating all efficient extreme points in the outcome set of a multiple objective linear programming problem. J. Global Optim., 13(1):1-24, 1998.

[2] D. Ciripoi, A. Löhne, and B. Weißing. Bensolve Tools - Calculus of Convex Polyhedra, Calculus of Polyhedral Convex Functions, Global Optimization, Vector Linear Programming for Octave and Matlab, Version 1.1. http: //tools.bensolve.org.

[3] D. Ciripoi, A. Löhne, and B. Weißing. A vector linear programming approach for certain global optimization problems. submitted to Journal of Global Optimization, 2017. http://arxiv.org/abs/1705.02297.

[4] O. Güler. Foundations of optimization. Springer, New York, NY, 2010.

[5] A. H. Hamel, A. Löhne, and B. Rudloff. Benson type algorithms for linear vector optimization and applications. J. Global Optim., 59(4):811-836, 2014.

[6] M. Herceg, M. Kvasnica, C. Jones, and M. Morari. Multi-Parametric Toolbox 3.0. In Proc. of the European Control Conference, pages 502-510, Zürich, Switzerland, July 17-19 2013. http://control.ee.ethz.ch/ mpt.

[7] F. Heyde and A. Löhne. Geometric duality in multiple objective linear programming. SIAM J. Optim., 19(2):836-845, 2008.

[8] N. Lauritzen. Undergraduate convexity. From Fourier and Motzkin to Kuhn and Tucker. Hackensack, NJ: World Scientific, 2013.

[9] A. Löhne. Vector optimization with infimum and supremum. Vector Optimization. Springer, Heidelberg, 2011.

[10] A. Löhne and B. Weißing. Bensolve - a Free Vector Linear Program Solver, Version 2.1. http://bensolve.org.

[11] A. Löhne and B. Weißing. Equivalence between polyhedral projection, multiple objective linear programming and vector linear programming. Mathematical Methods of Operations Research, 84(2):411-426, 2016. 
[12] L. Lovász and A. Schrijver. Matrix cones, projection representations, and stable set polyhedra. In Proceedings of DIMACS Workshop on Polyhedral Combinatorics 1989 (0), pages 1-17, 1990.

[13] R. Rockafellar. Convex Analysis. Princeton University Press, Princeton, 1972.

[14] B. Weißing. The Polyhedral Projection Problem. PhD thesis, Friedrich Schiller University Jena, 2017. 